\title{
Erratum: Ensemble inequivalence in the Blume-Emery-Griffiths model near a fourth-order critical point [Phys. Rev. E 100, 052135 (2019)]
}

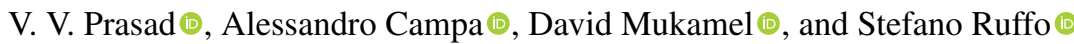 \\ (2) (Received 12 August 2021; published 1 September 2021)
}

DOI: 10.1103/PhysRevE.104.039901

We would like to correct a misprint in the paper. Equations (30) should be replaced by the following equations:

$$
\begin{aligned}
& K(\beta)=\frac{\beta}{\beta-1}-2 \ln (2 \beta-2)+\frac{2}{3}[\ln (2 \beta-2)]^{2}, \\
& \Delta(\beta)=[K(\beta)+\ln (2 \beta-2)] \beta^{-1}, \\
& \epsilon(\beta)=\frac{\Delta(\beta)}{2 \beta}+\frac{\ln (2 \beta-2)}{2 \beta^{2}} .
\end{aligned}
$$

The replaced equations were incorporated in the published paper by mistake. We stress that all calculations and figures presented in the paper were done with the correct expressions above, and are not affected by the misprint in the original paper. None of the results presented in the paper are affected by the misprint.

We thank Carlo Vanoni (SISSA, Italy) for pointing out the misprint. 